\title{
Research on Short-Term Wind Farm Output Power Prediction Model Based on Meteorological Data Collected by WSN
}

\author{
Li Ma ${ }^{1,2,3}$, Bo Li ${ }^{1,2}$, Du Jie ${ }^{1,2}$, Jian Shen ${ }^{1,2}$ and Jin Wang ${ }^{1,2}$ \\ ${ }^{1}$ Jiangsu Engineering Center of Network Monitoring, Nanjing University of \\ Information Science \& Technology, Nanjing 210044 \\ ${ }^{2}$ School of Computer \& Software, Nanjing University of Information \\ Science \& Technology, Nanjing 210044 \\ ${ }^{3}$ Key Laboratory of Meteorological Disaster of Ministry of Education, Nanjing \\ University of Information Science \& Technology, Nanjing 210044 \\ mali1775088@163.com;nmjnlxd@sina.com; wangjin@nuist.edu.cn
}

\begin{abstract}
The prediction of wind farm output power is considered as an effective way to increase the wind power capacity and improve the safety and economy of power system. It is one of the hot research topics on wind power. The wind farm output power is related to many factors such as wind speed, temperature, etc., which is difficult to be described by some mathematical expression. In this paper, Back Propagation (BP) neural network algorithm and genetic algorithm (GA) are combined to establish the prediction model of the short-term wind farm output power based on meteorological data collected by Wireless Sensor Network (WSN). The Meteorological data is used to determine the input variables of the BP neural network. Meanwhile, the GA is used to adjust the value of BP's connection weight and threshold dynamically. Then the trained BP neural network is used to predict the wind power. The experiment results show that our method has better prediction capability compared with that using BP neural network alone or using wind power formulas.
\end{abstract}

Key words: Wind Farm, Power Prediction, Artificial Neural Network, GA, WSN

\section{Introduction}

Wind energy is a kind of renewable and clean energy which has been concerned all over the world. Wind power is one of the fastest growing and most mature renewable power generation technologies [1]. However, wind power also has its drawbacks. Due to the properties of volatility, intermittency, low energy density, and uncontrollability, wind power is fluctuant and intermittent. When the proportion of wind power in power grid is small, the above characteristics are not able to influence the performance of power grid. However, as the development of wind power, the power generated from wind plays a very important role in power generation. Hence, in order to make a reasonable power generation plan and ensure the steady performance of power system, the prediction model of wind farm output power is required [2]. According to different standards, the methods of power prediction can be divided into different class. For example, 1) in accordance with the prediction time, the methods of power prediction are classified into long-term prediction method, mid-term prediction method, short-term prediction method and super short-term prediction method. 2) In accordance with the prediction parameters, the methods of power prediction are classified into wind speed-based prediction method and output power-based prediction method. 3) In 
accordance with the prediction models, the methods of power prediction are classified into physical prediction method, statistic prediction method, and learning method. In this paper, we use the neural network method to research the prediction of wind farm output power [3-4].

Recently, the technical of wind power prediction is not mature in China. Previous researches by continuous method, time sequence method, and neural network method make some improvement in practical experiment, without prediction accuracy [5-6]. Most of them lack of wind farm output power based on meteorological information collected from WSN. Furthermore, the prediction method on the basis of BP neural network using GA optimization is rarely mentioned. Therefore, in this paper, we design a new wind farm output power model, which is based on the meteorological data collected from WSN and BP neural network using GA optimization to perform the short-term wind power prediction. It is worth noting that short-term prediction can satisfy the requirement of market trade, system maintenance scheme, and safe power supply.

\section{Meteorological Data Collected by WSN}

Meteorological data can be collected by WSN. However, the way of data collection and transmission is manual setup or wired operation. When the number of wind fans in wind farm is huge, it will result in high communication cost and complicated route. It is not easy to operate. ZigBee is a wireless transmission technology with short distance, low speed, lower-power consumption, low cost, and low complexity. It works on the public frequency of 2.4 GHz. Moreover, the number of node in ZigBee network can reach 255 [7-8]. Therefore, in this paper, we use ZigBee-based technology to collect the meteorological data of wind farm fans. The steps of meteorological data collected by WSN is as follows: First, the meteorological data is collected by meteorological sensors. Secondly, the collected data is sent to processor by analog circuit, and then transmitted to data transmission module after AD transforming. Thirdly, data receiving module receives data and transmit it to Single Chip Microcomputer (SCM). Finally, the meteorological data is stored in SD card by SCM processing.

\section{Data and Methods}

\subsection{Data and the Number of Neural Network Input Variables Determination}

In this paper, we use the meteorological data collected by WSN to perform the short-term prediction, where the data is gather from Sep.1st to Sep.3rd in 2010 by No.5 fan in a wind farm from Hunan province. Note that the data is collected every 20 seconds, and averaged every 5 minutes from 15 selected values. It means that we can get 12 average values per hour. Finally, we receive 864 average values in three days in terms of wind speed, temperature, and power. We select the first 768 data of each group as training samples of neural network, while the last 96 data are treated as the prediction samples. First of all, the data of wind speed and temperature in neural network training samples is used as input. Power is used as output. When the training neural network is finished, the data of wind speed and temperature in prediction samples in the last 8 hours is used as input again, while the data of power is still used as output to compare the error.

\subsection{The Connection Weights and Thresholds of BP Neural Network Adjusted by GA}

The common method for the training of network connection weights and thresholds is BP neural network algorithm, which is a kind of algorithm based on error back propagation algorithm. Actually, it is a multilayer forward artificial neural network with supervisor 
training method. Furthermore, the BP neural network can approach nonlinear function with arbitrary accuracy. It has the ability of learning, self-adaption, and fault tolerance. Therefore, the constructed model has good robustness. The neuron of the BP neural network is denoted by a node, and the link vector between nodes is called weights vector. The basic structure of BP neural network contains three parts: the input layer, the hidden layer, and the output layer. The hidden layer includes one layer or multi layers, and neurons of different layer are connected by the connection weights [9-11]. Through the analysis of the previous part, we know that the input data contains speed and temperature. So the number of neurons in the input layer is 2, and the number of neurons in the output layer is 1, which is the wind power of wind farm. It is worth noting that the number of neurons in the hidden layer is related to the number of neurons in the input layer and output layer. The following formula (1) can be used as a reference, even through there is no detailed quantitative relationship.

$$
n_{1}=\sqrt{n+m}+a
$$

Where $n, m$, and $n_{1}$ denote the number of input, output, and hidden neurons respectively, and a means a constant value among 1 to 10 . Generally speaking, the value of input and output is fixed. We can get the different number of neurons of hidden layer by changing the value of $a$. In order to select the optimal number of hidden neurons, we can compare the performance of different neural networks with different number of neurons in the hidden layer [12]. According to the experiment result, we conclude that the number of neurons in the hidden layer is 5. Figure 1 shows the basic structure of BP neural network constructed by the above rules.

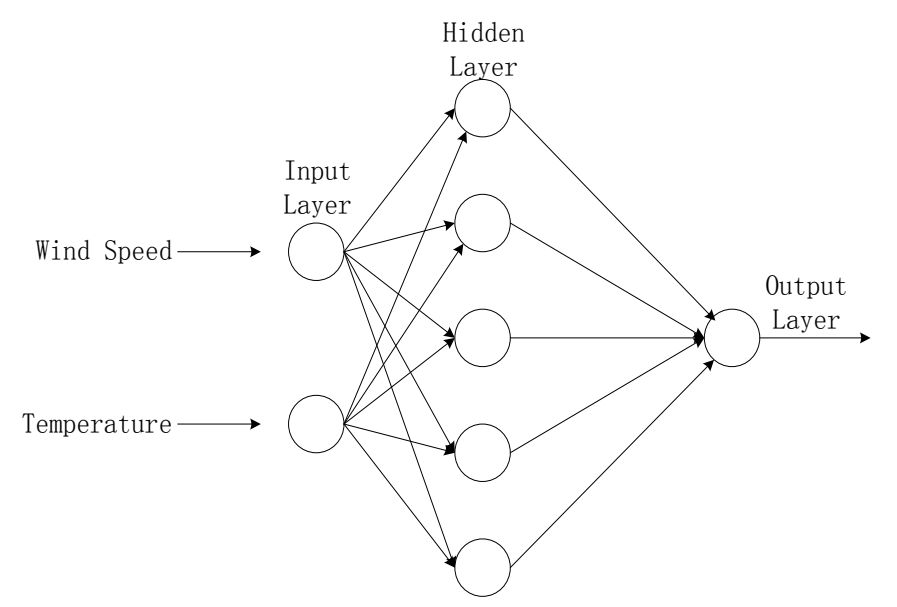

Figure 1. The Basic Structure of BP Neural Network

In addition, the data of the input layer must be normalized before training. The normalization method is a common data processing method before prediction, where all the data are transformed into the area from 0 to 1 . The purpose is to eliminate the order of magnitude differences among different dimension data, and to avoid the error of network prediction caused by the order of magnitude differences between the input and output data. The data normalization method used in this paper is called maximum minimum method, which is as follow.

$$
x_{k}=\frac{\left(x_{k}-x_{\min }\right)}{\left(x_{\max }-x_{\min }\right)}
$$


Where $x_{\min }$ is the minimum value among the data, and the $x_{\max }$ is the maximum value among the data.

The performance of BP neural network algorithm is very good, but the training speed is slow and the minimum value is sometime a local value. Note that GA is a global searching algorithm compared with the BP algorithm, the searching speed for optimal area of GA is faster [13]. Therefore, in this paper, we choose GA algorithm to optimize the BP neural network (GA-BP) to perform prediction.

Because the number of nodes in the input layer, output layer, and hidden layer is set as 2, 1 , and 5, the topology of BP neural network is 2-5-1, where there are 15 weights and 6 thresholds. Consequently, the code length of the initial individual is 21 , which is based on the real number coding method. In addition, the scale of the population also has affection on GA. Probably the scale of the population is chosen as 10 to 160 . In this paper, the scale of the initial population is 40 , according to the experiment result.

By the way, the fitness of GA is determined by the value of objective function. The objective function is presented as follow.

$$
F=\frac{1}{n}\left(\sum_{i=1}^{n}\left(y_{i}-o_{i}\right)^{2}\right)
$$

Where $n$ is the number of output training samples. $\gamma_{0}$ is expected output of the ith node in BP neural network, and $o_{i}$ is prediction output of the ith node. In this paper, we have 768 output training samples, so $n$ is 768 . We define the reciprocal of formula (3) as the value of individual fitness, which is as follow.

$$
f=768 /\left(\sum_{i=1}^{768}\left(y_{i}-o_{i}\right)^{2}\right)
$$

After determining the fitness function, we need to perform the selection operation. There are many methods for selection operation in GA such as roulette wheel method, tournament selection method, and so on. In this paper, we choose the roulette wheel method, which is based on the proportion of fitness. Each individual's selected probability $p_{i}$ is:

$$
p_{i}=f_{i} / \sum_{j=1}^{40} f_{j}
$$

Where 40 denotes the number of initial population.

After selection operation, the selected individual starts crossover operation. According to the experimental requirements, we set the probability of crossover is 0.4 . The probability of crossover will determine if the individual will perform crossover operation. Note that, the chromosome is selected at random, so is the crossover location. The chromosome crossover operation is as follow.

$$
\begin{aligned}
& \left.a_{k j}=a_{k j}(1-b)+a_{l j} b\right) \\
& \left.a_{l j}=a_{k j}(1-b)+a_{k j} b\right)
\end{aligned}
$$

Where $a_{k j p}$ is the kth chromosome, $a_{4 j}$ is the lth chromosome, and $b$ is a random number from $0-1$.

The last step in GA is mutation operation. The selection of mutation individual is similar to the crossover operation. According to the experimental requirements, we set the probability of mutation is 0.2 . The probability of mutation will determine if the individual will perform 
mutation operation. The chromosome is selected at random, so is the mutation location. The mutation operation is as follow.

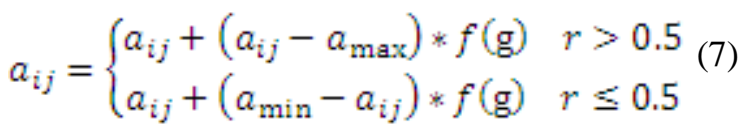

Where $a_{\max }$ is the upper bound of gene $a_{i j p}$ and $a_{\min }$ is the lower bound of gene $a_{i j}$. Note that $f(g)=r_{2}\left(1-g / G_{\max }\right)^{2}$, where $r_{2}$ is a random number, $g$ is the number of iterations, $G_{\max }$ is the maximum number of evolution, which is set as 50 , and $r$ is a random number from $0-1$.

We repeat the operations of selection, crossover, and mutation in different evolution sequences, and choose the best one from all the individuals. It will stop evolution when the number of evolution reaches the maximum or the objective function reaches the pre-defined value. At this time, the optimal values are set as the initial weights and thresholds of the BP neural network.

In addition, the choice of the hidden layer function and output layer function has a great influence on the prediction accuracy of BP neural network. In normal case, the transition function of node in the hidden layer is Logsig function or Tansig function, and the transition function of node in the output layer is Tansig function or Purelin function [14].

\section{Experiment Analysis}

Research shows that the wind power generated by wind fan in wind farm can be expressed as follow [15].

$$
\mathrm{P}=\frac{1}{2} C_{\mathrm{p}} A \rho v^{3}
$$

Where $C_{\mathrm{p}}$ is the power factor of the wind fan, $\rho$ is air density, $A$ is swept area of the wind wheel, and $v$ is wind speed. From the above formula, we can get the output power of wind fan is affected by wind speed. Hence, we define $\frac{1}{-} C_{\mathrm{p}} A \rho$ as a constant value. The constant value is denoted as CON. Then, we can get 768 value of CON from training samples by calculating the above expression (8). After that, the averaged value of CON can be obtained, which is 1.924652 . At last, we use 96 value of wind speed from prediction samples and the averaged value of $\mathrm{CON}$ to calculate 96 different value of wind power based on the expression (8).

Next, we analyze the predicated value of wind power from three different prediction methods, which are GA-BP method, simple BP method, and the method from expression (8). Figure 2 presents the comparison between the real value of wind power and the predicated value of wind power from the above three method. From the Figure 2, it shows that the method of expression (8) is the worst one, because the difference between the real value and the predicated value from expression (8) is the largest, compared with the difference between the real value and the predicated value from GA-BP and simple BP.

Figure 3 shows the relative errors calculated by the three methods. Note that, the real prediction error can be reflected by relative error. From the observation of Figure 3, we can get the average relative error is $14.59 \%$, and the maximum relative error is $49.45 \%$ by simple $\mathrm{BP}$. While the average relative error is $12.08 \%$, and the maximum relative error is $39.92 \%$ by GA-BP. Therefore, we can conclude that GA-BP algorithm is the best prediction method. 


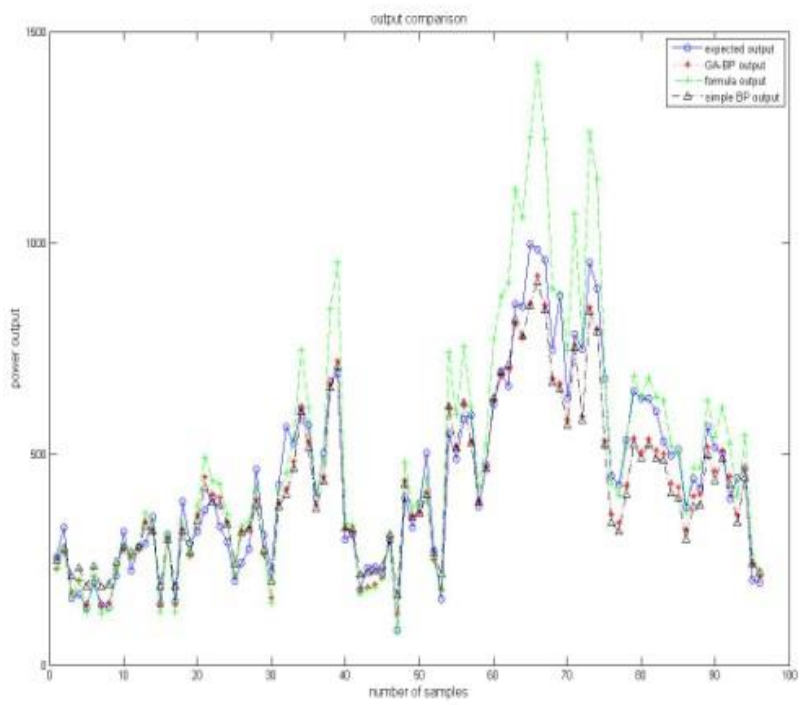

Figure 2. Output Comparison

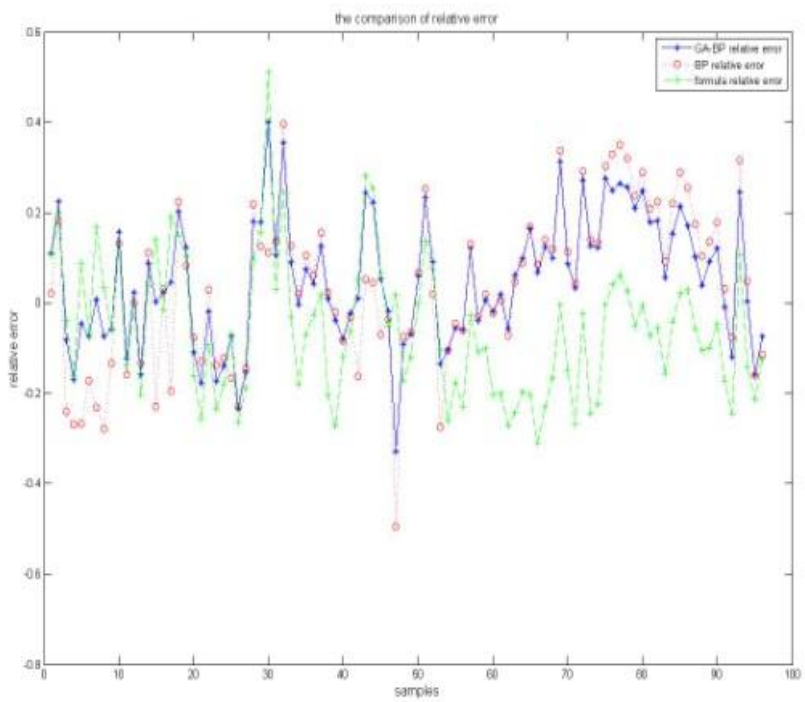

Figure 3. Relative Error Comparison

\section{Conclusions}

In this paper, we study the wind farm short-term power prediction method based on GA-BP algorithm. We use the meteorological data collected by WSN as the input and adjust the connection weights and thresholds of BP neural network by GA to obtain the optimal individual, and then perform BP neural network training. After that, we use the trained BP neural network to predict the output of wind power. The experiment results show that the GA-BP is better than the simple BP and the method from wind power equation. By the way, it 
avoids the fact that simple BP neural network fall in down local minimum value. In addition, it also resolves the slow convergence speed in simple BP. Hence, GA-BP can be used to predict the real wind power. It plays an important role in management of power grid system. In the future, we usually lack of real data. In order to establish the prediction model, we need to research the model established by the forecast data. Furthermore, we need to research whether the model established by the real data is suitable for the wind power prediction calculated by the forecast data.

\section{References}

[1] J. H. Huang and H. Peng, "The Prediction Research of Neural Network Method for Wind Farm Short-term Wind Power", Electrotechnics Electric, no. 9, (2009), pp. 57-60.

[2] G. F. Fan, W. S. Wang and C. Liu, "The Short-Term Wind Power Prediction System Based on Artificial Neural Network", Power System Technology, vol. 32, no. 22, (2008), pp. 72-76.

[3] Y. Q. Liu, S. Han and Y. S. Hu, "Review on Short-term Wind Power Prediction", Modern Electric Power, vol. 24, no. 5, (2007), pp. 6-11.

[4] L. J. Wang, X. Z. Liao and Y. Gao, "The Summarize for Power Model and Prediction Research of Wind Farm”, Power System Protection and Control, vol. 37, no. 13, (2009), pp. 118-121.

[5] M. Alexiadis, P. Dokopoulos, H. Sahsamanoglou and I. Manousaridis, "Short-term Forecasting of Wind Speed and Related Electrical Power", Solar Energy, vol. 63, no. 1, (1998), pp. 61-68.

[6] X. J. Yang, Y. Xiao and S. Y. Chen, "Wind Speed and Power Prediction Research of Wind Farm", Proceedings of The CSEE, vol. 25, no. 11, (2005), pp. 1-5.

[7] X. M. Li and Z. H. Zhao, "Study and experiment on ZigBee wireless sensor network", Electronic Measurement Technology, vol. 30, no. 6, (2007), pp. 133-136.

[8] F. C. Cao and G. M. Zhang, "Design of anemometric system based on ZigBee in wind farm", Renewable Energy Resources, vol. 29, no. 5, (2011), pp. 141-144.

[9] S. Haykin, "Neural Networks", China Machine Press, Beijing, (2004).

[10] M. T .Hagan, H. B. Demuth and M. H. Beale, "Neural Network Design", China Machine Press, Beijing (2002).

[11] Z. L. Jiang, "Introduction to Artificial Neural Networks", Higher Education Press, Beijing, (2001).

[12] L.Q. Han, "Artificial Neural Network theory, Design and Application”, Chemical Industry Press, Beijing (2011).

[13] W. Fu, X. Y. Yang and W. Feng, "Wind Speed Forecasting Using Genetic Neural Model", Modern Scientific Instruments, no. 6, (2009), pp. 48-52.

[14] F. Shi, X. C. Wang and L. Yu, "Analysis of Thirty Cases about MATLAB Neural Network", Bei Hang University Press, Beijing, (2011).

[15] C. Liu, G. F. Fan and W. S. Wang, "A Combination Forecasting Model for Wind Farm Output Power", Power System Technology, vol. 33, no. 13, (2009), pp. 74-79.

\section{Authors}

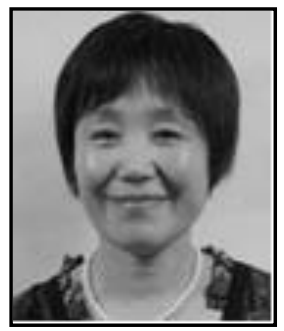

\section{Li MA}

She received her B.S. degree in 1985 from the Chengdu Institute of Meteorology and her Ph. D degree in 2011 from Nanjing University of Information Science and Technology. She is a professor and tutor for graduates in Nanjing University of Information Science and Technology. Her main research interests include image processing, pattern recognition, and meteorological information processing and data assimilation. 


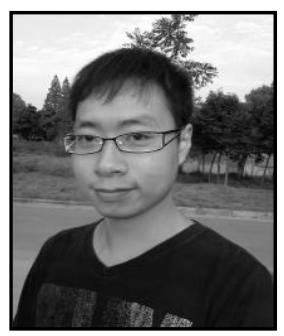

\section{Bo Li}

He was born on May 12, 1987. Currently $\mathrm{He}$ is a master candidate in Nanjing University of Information Science and Technology. He received his bachelor degree in Chongqing Normal University.His areas of interest are short-term wind power prediction, meteorological information processing and data assimilation.

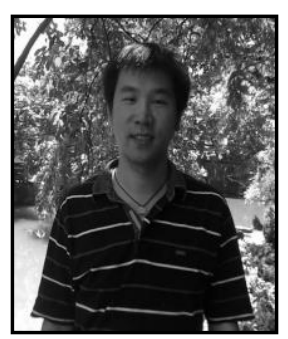

\section{Jie Du}

He was born on July 20, 1978. He is an associate professor and master tutor in Nanjing University of Information Science and Technology. He received his $\mathrm{B} \mathrm{S}$, master, $\mathrm{Ph}$. D degree from Nanjing University of Technology. He worked in the college of electrical engineering, Zhejiang University mobile post-doctoral stations. His main research interests include short-term wind power prediction, nonlinear time series intelligent prediction method, neural network, data mining and genetic algorithm.

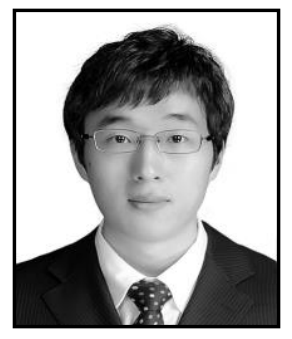

\section{Jian Shen}

He received the B.E. degree from Nanjing University of Information Science and Technology, Nanjing, China, in 2007 and the M.E. and Ph.D. degrees in Computer Science from Chosun University, Gwangju, Korea, in 2009 and 2012, respectively. Since late 2012, he has been an appointed professor in the School of Computer and Software at Nanjing University of Information Science and Technology, Nanjing, China. His research interests include computer networking, security systems, mobile computing and networking, ad hoc networks and systems, and ubiquitous sensor networks.

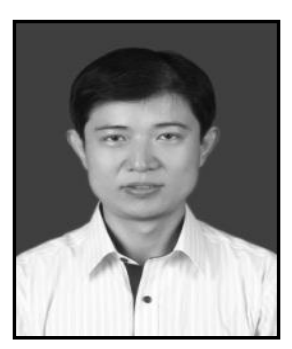

\section{Jin Wang}

He received the B.S. and M.S. degree in the Electronical Engineering from Nanjing University of Posts and Telecommunications, China in 2002 and 2005, respectively. He received Ph.D. degree in the Ubiquitous Computing laboratory from the Computer Engineering Department of Kyung Hee University Korea in 2010. Now, he is a professor in the Computer and Software Institute, Nanjing University of Information Science and technology. His research interests mainly include routing protocol and algorithm design, performance evaluation and optimization for wireless ad hoc and sensor networks. He is a member of the IEEE and ACM. 